\title{
Hydantoin analogs inhibit the fully assembled ClpXP protease without affecting the individual peptidase and chaperone domains
}

\author{
Christian Fetzer, ${ }^{a}$ Vadim S. Korotkov ${ }^{a, b}$ and Stephan A. Sieber ${ }^{a,+}$
}

Proteolysis mediated by ClpXP is a crucial cellular process linked to bacterial pathogenesis. The development of specific inhibitors has largely focused on ClpP. However, this focus was challenged by a recent finding showing that conformational control by ClpX leads to a rejection of ClpP binders. Thus, we here follow up on a hit molecule from a high throughput screen performed against the whole ClpXP complex and demonstrate that stable inhibition with high potency is possible. Further investigations revealed that the small molecule binds to ClpP without affecting its activity. Likewise, the molecule does not inhibit ClpX and retains the overall oligomeric state of ClpXP upon binding. Structure activity relationship studies confirmed structural constraints in all three parts of the molecule suggesting binding into a defined stereospecific pocket. Overall, the inhibition of ClpXP without affecting the individual components represents a novel mechanism with perspectives for further optimization for in situ applications. Caseinolytic protease $\mathrm{P}(\mathrm{ClpP})$ is a tetradecameric enzyme belonging to the serine hydrolase family. ${ }^{1,2}$ The enzyme is able to digest small peptides but cannot act on larger protein substrates by itself. In the cell ClpP binds to cognate chaperones such as ClpX via both apical sites of the tetradecameric barrel forming the proteolytically active CIpXP complex. ${ }^{3}$ Within this complex ClpX recognizes proteins prone to degradation, e.g. during cellular stress, unfolds them under consumption of ATP and finally pushes the peptide chain into the ClpP barrel for subsequent proteolytic digest. ClpXP activity has been shown to be crucial for cell homeostasis in cancer cells as well as for the control of virulence in pathogenic bacteria such as Staphylococcus aureus. ${ }^{4,5}$ As a consequence of these functions the genetic deletion as well as chemical inhibition led to positive therapeutic effects in treated leukemia as well as bacterial cells, respectively. ${ }^{5-7}$ However, major challenges in the development of inhibitors are the selectivity of compounds for ClpP or ClpX, their stability in plasma, residence time when bound to the enzyme as well as conformational control of ClpX over ClpP resulting in rejection of compounds. ${ }^{8,9}$ One of the reasons for these limitations was the previous focus on solely ClpP during inhibitor development and just recent mechanistic insights unraveled the importance of the whole ClpXP complex as more suitable target to mimic cellular activity. In order to overcome the limitations of previous covalent inhibitors ${ }^{10}$ such as betalactones, phenyl esters and boronic acids as well as reversible inhibitors such as oxazoles, a new high-throughput screening (HTS) campaign utilized an assay monitoring the activity of whole SaClpXP complex. ${ }^{11}$ The activity was monitored by the degradation of green fluorescent protein (GFP) tagged with a SsrA peptide sequence, required for ClpX mediated recognition. ${ }^{12}$ In principle, this HTS could deliver 3 types of inhibitors: 1) inhibitors of ClpP, 2) inhibitors of ClpX and 3) inhibitors that block the interplay between ClpX and ClpP. The HTS reveled 6 validated hits which were all able to potently inhibit the whole proteolytic complex. One inhibitor was selected for follow up studies demonstrating that it acted solely on the ClpX chaperone. ${ }^{11}$ Binding of this compound to ClpXP led to a disruption of the oligomeric complex associated with a disassembly of ClpX hexamers into monomers. Correspondingly, the compound inhibited ClpX ATPase activity but had no effect on ClpP.

Here, we follow up on these HTS results and introduce, in addition to already existing ClpP and ClpX inhibitors, an unprecedented compound which neither influences ClpP nor ClpX activity but potently blocks turnover of the whole complex. Synthesis of several derivatives reveals insights into the structure activity relationship (SAR) and the preparation of two enantiomers shows a clear preference of one enantiomer for complex inhibition. Together with thermal shift assays, these

\footnotetext{
a. Technische Universität München, Center for Integrated Protein Science Munich

(CIPSM), Lichtenbergstraße 4, 85748 Garching (Germany)

b. Current address: Helmholtz-Zentrum für Infektionsforschung, Inhoffenstraße 7,

38124 Braunschweig (Germany)

+E-Mail: stephan.sieber@tum.de
} 
results suggest the existence of a defined, stereospecific binding pocket located on ClpP.
A)

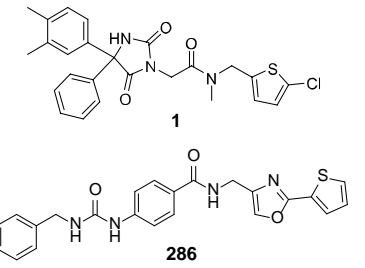

C)

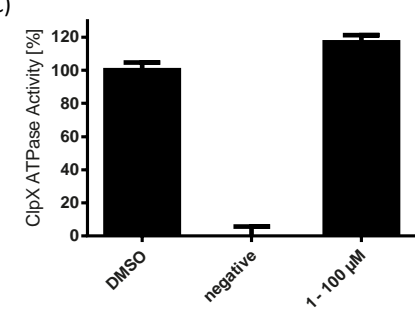

B)

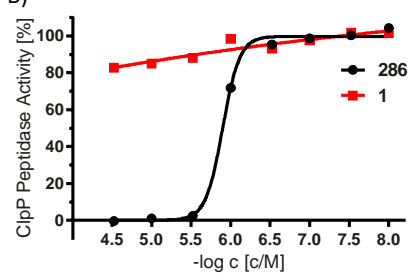

D)

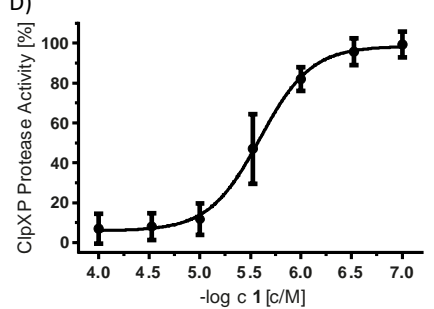

Figure 1 Influence of compound 1 on creatine kinase, ClpP, ClpX and ClpXP activity. A) Chemical structure of ClpXP inhibitor 1. B) 1 does not alter ClpP peptidase activity in a fluorescent assay ( $1 \mu \mathrm{M}$ ClpP; mean \pm standard error). The non-covalent ClpP inhibitor 286 was used as a positive control. C) ClpX ATPase activity assay is not inhibited by 1 (100 $\mu \mathrm{M}$ final compound concentration; mean \pm standard deviation). D) 1 shows a dosedependent inhibition of the ClpXP protease (mean \pm standard error).

Compound $\mathbf{1}$ was identified as one of the most potent inhibitors of ClpXP activity in a previous HTS (Figure $1 \mathrm{~A}$ ). ${ }^{11}$ Prior to further mechanistic studies of this compound, we performed a secondary assay to exclude a putative activity against creatine kinase, an enzyme required in the HTS to regenerate ATP from ADP in the GFP assay. In contrast to a positive control with iodoacetamide, no significant reduction of activity could be observed in the Kinase-Glo assay (Supporting Figure 1). Interestingly, $\mathbf{1}$ did neither inhibit ClpP peptidase activity, as measured by turnover of a fluorescent substrate (positive control with a previous oxazole inhibitor), ${ }^{9}$ nor ClpX mediated ATP hydrolysis (Figure 1B, C). However, when ClpXP activity was tested via the GFP degradation assay, the compound revealed potent inhibition with an $\mathrm{IC}_{50}$ of $1.9 \mu \mathrm{M}$, a remarkable activity when considering that $4 \mu \mathrm{M}$ of enzyme (ClpP monomer) is needed in this assay (Figure 1D).

To further localize if the compound binds to ClpP or ClpX we performed thermal shift assays in the presence or absence of 1. A strong stabilization as indicated by a $9 \mathrm{~K}$ shift in melting temperature was observed for ClpP but not ClpX (Figure 2A). As expected by the structure of $\mathbf{1}$ which lacks obvious electrophilic moieties, no covalent modification was observed on ClpP via mass spectrometry analysis (Figure 2B). Analytical size exclusion chromatography showed that $\mathbf{1}$ does neither deoligomerize ClpP nor ClpX as well as the whole complex (Figure $2 \mathrm{C}$ ). This indicates an unprecedented mode of inhibition of ClpXP via reversible binding to solely ClpP while retaining the overall oligomeric assembly.

Subsequent SAR studies aimed to decipher the most important structural elements of 1 responsible for inhibition. For this the compound was dissected into three parts, namely the hydantoin, linker and thiophene moieties (Figure 3A). All analogs were either synthesized or obtained from commercial
A)
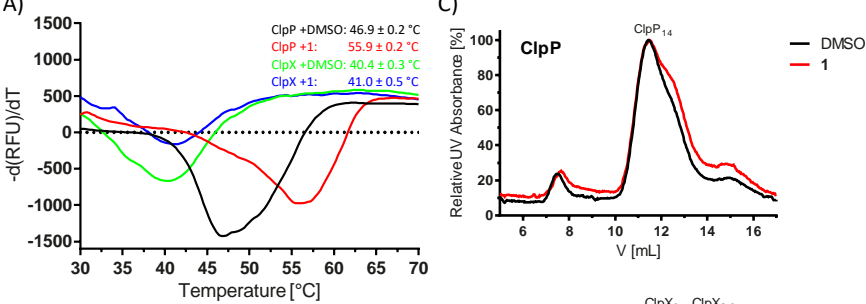

B)
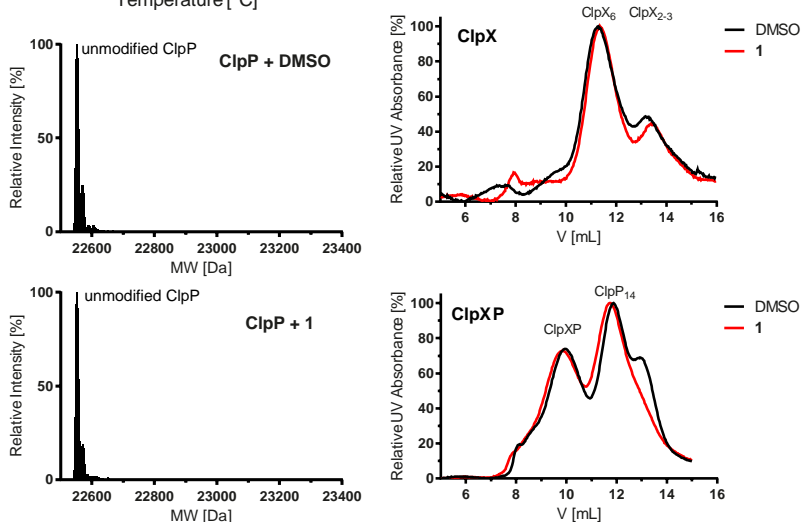

Figure 2 Interaction of 1 with $\mathrm{ClpP}$ and $\mathrm{ClpX}$. A) Thermal-shift assay depicts strong stabilization of $\mathrm{ClpP}$ while the melting point of $\mathrm{ClpX}$ remains unaltered. Experiments were conducted with $10 \mu \mathrm{M}$ enzyme concentration in PBS buffer and DMSO or $60 \mu \mathrm{M}$ of 1 . B) No covalent modifications of $\mathrm{ClpP}$ are detectable by intact-protein mass spectrometry upon treatment with 1 at $100 \mu \mathrm{M}$ (100-fold excess). C) Size-exclusion chromatography experiments show no substantial change of ClpP-tetradecamer, ClpX-hexamer and ClpXP-complex upon treatment with $100 \mu \mathrm{M} 1$.

sources and their corresponding structures are displayed in Figure 3B. The synthesis started with reductive amination of substituted thiencarbaldehyde with alkylamines (Scheme 1). The obtained secondary amines were acylated with 2bromoacetyl bromide. 5,5-Diarylhydantoins were synthesized from the corresponding 1,2-diaryl-1,2-diones and urea. Regioselective $\mathrm{N}$-alkylation of 5,5-diarylhydantoins with 2-

$$
\text { 2: } \mathrm{R}_{1}=\mathrm{Ph}_{\mathrm{R}}
$$

Scheme 1 Synthesis of 1 analogs. Reagents and Conditions: $a$ ) urea, $40 \%$ aq. $\mathrm{NaOH}, \mathrm{EtOH}$ reflux, $3 \mathrm{~h}$, then $\mathrm{HCl}$, r. t., $41-66 \%$; b) $\mathrm{R}_{2} \mathrm{NH}_{2}$, $\mathrm{HOAc}$, THF, $\mathrm{MeOH}, 0^{\circ} \mathrm{C}, 4 \mathrm{~h}$, then r. t., 18 h, 92-94\%; c) $\mathrm{NaBH}_{4}$, EtOH, 2 h, r. t., $86-91 \%$; d) 2-bromoacetyl bromide, $\mathrm{CH}_{2} \mathrm{Cl}_{2}, 0^{\circ} \mathrm{C}$ to r. t., $1 \mathrm{~h}, 38-76 \%$; e) $\mathrm{Cs}_{2} \mathrm{CO}_{3}$, DMF, r. t., $3 \mathrm{~h}, 25-57 \%$ 
bromoacetamides using $\mathrm{Cs}_{2} \mathrm{CO}_{3}$ as a base led to the desired products. ${ }^{13}$ Alterations in the thiophene section of the molecule by exchange of the halogen substituted thiophene with a phenyl group (2) resulted in a tenfold lower $\mathrm{IC}_{50}$ value in the ClpXP protease assay (Figure $3 \mathrm{C}$ ). Propylation (3) instead of methylation (4) of the nitrogen located in the linker almost completely prevented inhibition. However, shortening of the linker and removal of the nitrogen (5) still retained some activity, although no full inhibition could be obtained. Replacement of one phenyl group by a methyl substituent at the hydantoin core structure (6) resulted in a 40-fold drop in potency. This suggests that sterically demanding groups are necessary for potent inhibition. Hence, the role of additional methyl substituted phenyl groups was further elucidated. Disubstituted compound $\mathbf{1}$ exhibited the highest potency while compound $\mathbf{7}$, bearing two equally methyl substituted phenyl moieties, showed complete loss of activity. This result indicates that only one enantiomer of $\mathbf{1}$ is binding to ClpP as the methyl substituent seems to clash with the binding site in one of the two possible conformations. Consequently, enantiomers were separated by chiral high-performance liquid chromatography. Due to the small amount of isolated enantiomers the absolute configuration could not be assigned by $\mathrm{X}$-ray analysis.

Individual testing of both enantiomers in the ClpXP protease assay revealed that only 1-E1 showed inhibition, while the other enantiomer (1-E2) was completely inactive (Figure 3C). This suggests the existence of a defined, stereospecific and druggable binding pocket enabling further studies to improve the in situ activity via parameters such as uptake and stability. Studies into virulence were performed via Western blot analysis and LC-MS/MS. Only a slight reduction of alpha-hemolysin, a major virulence factor linked to ClpXP activity, was observed for the racemate and 1-E2 but not for 1-E1 treated samples (Supporting Figure 2). However, secretome analysis of 1 enantiomers by LC-MS/MS showed no change of alphahemolysin compared to DMSO treated samples (Supporting Table 1). We thus conclude that physicochemical parameters need to be optimized for more effective studies on whole cells. A)

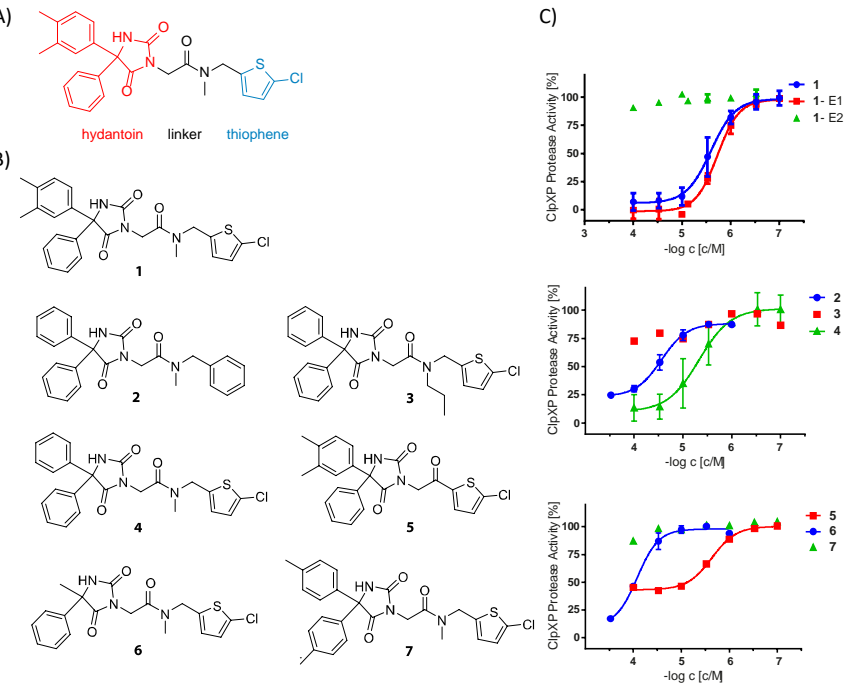

Figure 3 Structure-activity relationship studies of 1 analogs in ClpXP-protease assay. A Dissection of 1 into 3 parts. B) Chemical structures of $\mathbf{1}$ analogs. C) Inhibition data of all compounds in the ClpXP protease assay.

\section{Conclusions}

In conclusion, 1 constitutes a new class of ClpXP inhibitors that neither act on peptidase nor chaperone activities but requires the whole proteolytic complex for impairing its substrate turnover. The compound binds stereospecifically into a yet unidentified binding pocket on $\mathrm{ClpP}$, which according to our SAR data exhibits a defined shape allowing only restricted modifications. The elucidation of this pocket in future studies may further unravel the precise mechanism of action and guide the design of improved inhibitors also bearing in situ activities.

\section{Conflicts of interest}

There are no conflicts to declare.

\section{Acknowledgements}

This work was supported by the Deutsche Forschungsgemeinschaft SI1096/8-1 (ClpP) and CIPSM.

\section{Notes and references}

1. R. T. Sauer and T. A. Baker, Annu. Rev. Biochem., 2011, 80, 587-612.

2. K. Liu, A. Ologbenla and W. A. Houry, Crit. Rev. Biochem. Mol. Biol., 2014, 49, 400-412.

3. T. A. Baker and R. T. Sauer, Biochim. Biophys. Acta, 2012, 1823, 15-28.

4. D. Frees, S. N. Qazi, P. J. Hill and H. Ingmer, Mol. Microbiol., 2003, 48, 1565-1578.

5. D. Frees, K. Sorensen and H. Ingmer, Infect. Immun., 2005, 73, 8100-8108.

6. A. Cole, Z. Wang, E. Coyaud, V. Voisin, M. Gronda, Y. Jitkova, R. Mattson, R. Hurren, S. Babovic, N. Maclean, I. Restall, X. Wang, D. V. Jeyaraju, M. A. Sukhai, S. Prabha, S. Bashir, A. Ramakrishnan, E. Leung, Y. H. Qia, N. Zhang, K. R. Combes, T. Ketela, F. Lin, W. A. Houry, A. Aman, R. Al-Awar, W. Zheng, E. Wienholds, C. J. Xu, J. Dick, J. C. Wang, J. Moffat, M. D. Minden, C. J. Eaves, G. D. Bader, Z. Hao, S. M. Kornblau, B. Raught and A. D. Schimmer, Cancer cell, 2015, 27, 864-876.

7. T. Böttcher and S. A. Sieber, J. Am. Chem. Soc., 2008, 130, 14400-14401.

8. M. W. Hackl, M. Lakemeyer, M. Dahmen, M. Glaser, A. Pahl, K. Lorenz-Baath, T. Menzel, S. Sievers, T. Bottcher, I. Antes, H. Waldmann and S. A. Sieber, J. Am. Chem. Soc., 2015, 137, 8475-8483.

9. A. Pahl, M. Lakemeyer, M. T. Vielberg, M. W. Hackl, J. Vomacka, V. S. Korotkov, M. L. Stein, C. Fetzer, K. LorenzBaath, K. Richter, H. Waldmann, M. Groll and S. A. Sieber, Angew. Chem. Int. Ed., 2015, 54, 15892-15896.

10. F. Ye, J. Li and C. G. Yang, Mol. Biosyst., 2016, 13, 23-31.

11. C. Fetzer, V. S. Korotkov, R. Thanert, K. M. Lee, M. Neuenschwander, J. P. von Kries, E. Medina and S. A. Sieber, Angew. Chem. Int. Ed., 2017, 56, 15746-15750.

12. S. Gottesman, E. Roche, Y. Zhou and R. T. Sauer, Genes Dev., 1998, 12, 1338-1347.

13. N. T. Tzvetkov, H. Euler and C. E. Müller, Beilstein J. Org. Chem., 2012, 8, 1584-1593. 